EESTI NSV TEADUSTE AKADEEMIA TOIMETISED. 28. KOIDE

FOUSIKA * MATEMAATIKA. 1979, NR. 3

ИЗВЕСТИЯ АКАДЕМИИ НАУК ЭСТОНСКОИ ССР. ТОМ 28 ФИЗИКА * МАТЕМАТИКА. 1979, № 3

\title{
РЕШЕНИЕ ЗАДАЧ ЦЕЛОЧИСЛЕННОГО ПРОГРАММИРОВАНИЯ С ДОПОЛНИТЕЛЬНЫМИ АЛЬТЕРНАТИВНЫМИ УСЛОВИЯМИ
}

(Представлена А. Хумалом)

\section{§1. Постановка задачи}

В [ $\left.{ }^{1}\right]$ рассматривалась задача целочисленного программирования при дополнительном условии, что в каждой заданной группе линейных неравенств выполняется по крайней мере одно. Предложенная в настоящей работе постановка проблемы позволяет охватить задачи с более широким классом альтернативных условий, нежели в $\left[{ }^{1}\right]$.

Пусть $B$ обозначает множество индексов базисных переменных, $N$ - множество индексов небазисных переменных и пусть $B \cup N=$ $=\{1,2, \ldots, n\}$.

Расомотрим задачу: максимизировать функцию

$$
x_{0} \equiv a_{00}+\sum_{j \in N} a_{0 j}\left(-x_{j}\right)
$$

при ограничениях

$$
\begin{array}{ll}
x_{i} \equiv a_{i 0}+\sum_{j \in N} a_{i j}\left(-x_{j}\right) \geqslant 0, & i \in B, \\
x_{j} \equiv-\left(-x_{j}\right) \geqslant 0, & j \in N, \\
x_{j} \text { - целое при } j \in N &
\end{array}
$$

и дополнительном условии, что выполняются или заданные ограничения

$$
\begin{aligned}
& x_{p} \equiv a_{p 0}+\sum_{j \in N} a_{p j}\left(-x_{j}\right) \geqslant 0, \\
& p=n+1, n+2, \ldots, n+l_{1},
\end{aligned}
$$

или ограничения

$$
\begin{aligned}
& x_{q} \equiv a_{q 0}+\sum_{j \in N} a_{q j}\left(-x_{j}\right) \geqslant 0, \\
& q=n+l_{1}+1, \ldots, n+l_{1}+l_{2},
\end{aligned}
$$

или ограничения

$$
\begin{aligned}
x_{s} & \equiv a_{s 0}+\sum_{j \in N} a_{s j}\left(-x_{j}\right) \geqslant 0, \\
s & =n+l_{1}+\ldots+l_{t-1}+1, \ldots, n+l_{1}+\ldots+l_{t},
\end{aligned}
$$


где $t-$ число групп альтернативных ограничений $(5 \mathrm{a})-(5 \kappa)$. Такая задача является, вообще говоря, многоэкстремальной.

Предполагаем, что все коэффициенты $a_{i j}$ в выражениях (1), (2) и дополнительных условиях (5a) - $(5 \kappa)$ - целые числа. Тогда и переменные $x_{i}$, где $i \notin N$, - целые и рассматриваемая задача полностью целочисленная.

Альтернативные условия (5a) $\vee(56) \bigvee \ldots \bigvee(5 \kappa)$ в дальнейшем будем называть условиями (5) и рассматриваемую задачу - задачей (1) -(5). При $l_{1}=l_{2}=\ldots=l_{t}=1$ она сводится к задаче, рассмотренной в [ $\left.{ }^{1}\right]$.

\section{$\S 2$. Построение правильного отсечения}

Базисное решение системы линейных уравнений (2), (5) (без ограничения неотрицательности переменных)

$$
x_{j}=0 \text { при } j \in N, x_{i}=a_{i 0} \text { при } i \notin N
$$

является, вообще говоря, псевдопланом задачи (1)-(5). Предположим, что этот псевдоплан не удовлетворяет альтернативным условиям (5), т. е. в каждой группе ограничений $(5 \mathrm{a}),(56), \ldots,(5 \kappa)$ не выполняется по крайней мере одно. Это значит, что существуют индексы $p \in\left\{n+1, \ldots, \quad n+l_{1}\right\}, \quad q \in\left\{n+l_{1}+1, \ldots, n+l_{1}+l_{2}\right\}, \ldots$, $s \in\left\{n+l_{1}+\ldots+l_{t-1}+1, \ldots, n+l_{1}+\ldots+l_{t}\right\}$, при которых

$$
a_{p 0}<0, a_{q 0}<0, \ldots, a_{s 0}<0 .
$$

Но каждое допустимое решение задачи (1)-(5) (и задачи (1)-(3), (5)) удовлетворяет всем ограничениям по крайней мере одной из групп условий (5). Это, в частности, означает, что каждое допустимое решение удовлетворяет по крайней мере одному из неравенств

$$
x_{r} \equiv a_{r 0}+\sum_{j \in N} a_{r j}\left(-x_{j}\right) \geqslant 0, \quad r \in R,
$$

где $R=\{p, q, \ldots, s\}$ - множество индексов неравенств (7). По необходимому условию (8) построим правильное отсечение для задачи (1) - (5). Вне зависимости от того, при каком индексе $r \in R$ оно имеет место, в силу отрицательности $a_{r 0}$ имеем

$$
0 \leqslant-1+\sum_{j \in N}\left(a_{r j} / a_{r 0}\right) x_{j} \leqslant-1+\sum_{j \in N} \max _{r \in R}\left(a_{r j} / a_{r 0}\right) x_{j} .
$$

Обозначив

$$
d_{j}=\max _{r \in R}\left(a_{r j} / a_{r 0}\right)
$$

получим

$$
-1+\sum_{j \in N}\left(-d_{j}\right)\left(-x_{j}\right) \geqslant 0 .
$$

Согласно построению, неравенство (10) выполняется при каждом допустимом решении задачи (1)-(5) (а также задачи (1)-(3), (5)), но не выполняется при псевдоплане (6), где $x_{j}=0, j \in N$. Следогательно, оно и является искомым отсечением. Как известно ([$\left.{ }^{2}\right]$, с. 192), из неравенства (10) можно получить новое правнльное отсеченпе с целыми коэффициентами

$$
x_{u} \equiv[-1 / \lambda]+\sum_{j \in N}\left[-d_{j} / \lambda\right]\left(-x_{j}\right) \geqslant 0, \quad x_{u}-\text { целое }
$$


где $\lambda$ - положительное число. Отсечение (11), где $\lambda$ выбирается подходящим образом, используется в приводимом ниже алгоритме.

\section{§ 3 . Конечный алгоритм для решения поставленной задачи}

Рассмотрим задачу (1)-(5). Для $j=0$ и $j \in N$ обозначим

$$
A_{j}=\left(a_{0 j}, a_{1 j}, \ldots, a_{n j}\right)^{\mathrm{T}}
$$

и

$$
\bar{A}_{j}=\left(a_{0 j}, \ldots, a_{n j}, a_{n+1, j}, \ldots, a_{n+l_{1}+\ldots+l_{t, j}}\right)^{\mathrm{T}},
$$

где $a_{i j}=0$ при $i \in N(i \neq j)$ и $a_{j j}=-1(j \in N)$, а знак «Т» означает транспонирование.

Предположим, что исходная симплексная таблица

$$
A=\underbrace{\left(\bar{A}_{0}, \ldots, \bar{A}_{j}, \ldots\right)}_{1+|N| \text { столбцов }} \quad(j \in N)
$$

является $l$-нормальной, целевая функция (1) ограничена снизу на множестве допустимых решений и $a$ - ее нижняя граница. Тогда на основании полностью целочисленного (третьего) алгоритма Гомори $\left[{ }^{2,3}\right]$ с учетом модификации, предложенной в $\left[{ }^{2}\right]$ (с. 184-186), получим для решения задачи (1)-(5) алгоритм, аналогичный алгоритму из [ $\left.{ }^{1}\right]$. Изложим его.

1. Проверяем выполнимость условия $a_{00} \geqslant a$. При положительном ответе переходим к п. 2, при отрицательном - задача неразрешима.

2. Проверяем наличие отрицательных среди $a_{i 0}, i \in B$. Если их нет, переходим к п. 3. Если имеются, выбираем первое из них, пусть это будет $a_{k 0}$, и проверяем, есть ли среди коэффициентов $a_{k j}$ отрицательные. Если нет - задача неразрешима. Если существуют, строим по строке с индексом $k$ отсечение полностыо целочисленного алгоритма Гомори. Переходим к п. 4.

3. Проверяем наличие группы альтернативных условий (5), для которой все свободные члены нестрицательные. Если такая группа найдется, то базисное решение (6) оптимально. Если ее нет, выбираем из каждой группы (5a), (5б), ..., (5к) опраничение с отрицательным свободным членом и строим отсечение $(10)$. Если все $-d_{j} \geqslant 0$, то альтернативные условия противоречивы и задача неразрешима. Если имеются $-d_{j}<0$, строим отсечение (11), где $\lambda$ определяется по правилам полностью целочисленного алгоритма Гомори. Переходим к п. 4.

4. Принимаем построенное отсечение за ведущую строку и выполняем симплексную итерацию. При этом, если полученное в пп. 2 или 3 отсечение имеет вид

$$
x_{u} \equiv a_{u 0}+\sum_{j \in N} a_{u j}\left(-x_{j}\right) \geqslant 0, \quad x_{u}-\text { целое }
$$

$\left(u>n+l_{1}+\ldots+l_{t}\right)$, а $l-$ индекс ведущего столбца, переход к новой симплексной таблице $A^{*}$ осуществляется по формулам

$$
\bar{A}_{u}^{*}=\bar{A}_{l}, \quad \bar{A}_{j}^{*}=\bar{A}_{j}+a_{u j} \bar{A}_{l} \quad(j \neq u),
$$

причем множества базисных и небазисных индексов изменяются следующим образом

$$
B^{*}=B \cup\{l\}, \quad N^{*}=N \cup\{u\} \backslash\{l\},
$$


а число переменных увеличивается на одно. После симплексной итерации коэффициенты прибавленного отсечения вычеркиваем.

5. Матрицу $A^{*}$, множества $B^{*}$ и $N^{*}$ обозначаем теперь через $A, B$ и $N$ соответственно и выбираем за текущие. Задача опять принимает вид (1)-(5). Переходим к п. 1.

Для доказательства конечности алгоритма используем лексикографическое неравенство

$$
A_{0}^{*}=A_{0}+a_{u 0} A_{l}<A_{0},
$$

которое, в силу $a_{u 0}<0$ и $A_{l}>0$, вытекает из (14) при $j=0$. Следуя рассуждениям, используемым для доказательства конечности полностыо целочисленного алгоритма Гомори $\left({ }^{3}\right]$, с. 303$)$, приходим после конечного числа симплексных итераций к выводу, что задача либо не имеет решения, либо вектор $A_{0}$ уже не изменяется, причем все $a_{i 0} \geqslant 0$ при $i \in B$. Но тогда выполняются и альтернативные условия. Действительно, в противном случае следовало бы построить отсечение (10) и после выполнения пп. 3 и 4 рассматриваемого алгоритма мы получили бы $A_{0}{ }^{*} \prec A_{0}$.

П и м ечание. Изложенный алгоритм применим и для задач с несколькими системами альтернативных условий (5) (в каждой из которых должна выполняться одна группа ограничений). К такой же задаче приводится задача типа (1)-(5), в которой вместо одной группы ограничений требуется выполнение по крайней мере $k$ групп $(1<k<t)$. Легко доказать, что для этого необходимо и достаточно, чтобы в каждой подсистеме с $t-(k-1)$ группами ограничений выполнялась по крайней мере одна.

\section{§4. Численный пример}

Для иллюстрации алгоритма рассмотрим следующую конкретную задачу: максимизировать линейную форму

$$
x_{1}+x_{2}
$$

при условиях

$$
\begin{gathered}
x_{1}+x_{2} \leqslant 10, \\
x_{1} \geqslant 0, x_{2} \geqslant 0, x_{1}, x_{2} \text { - целые, }
\end{gathered}
$$

и дополнительном условии, чтобы из трех групп неравенств

или $\left\{\begin{array}{r}-2 x_{1}+x_{2} \leqslant 0, \\ x_{1} \leqslant 2\end{array}\right.$ или $\left\{\begin{array}{r}-x_{1}+x_{2} \leqslant 0, \\ x_{1} \leqslant 4\end{array}\right.$ или $\left\{\begin{array}{r}-x_{1}+2 x_{2} \leqslant 0, \\ x_{1} \leqslant 6\end{array}\right.$

выполнялась по крайней мере одна.

Графически легко проверить, что область допустимых решений соответствующей непрерывной задачи не является выпуклой и содержит три точки локального максимума. Решение примера представлено в табл. $1-7$, где $x_{0}, x_{3}, \ldots, x_{9}-$ вспомогательные неотрицательные (кроме $x_{0}$ ) целочисленные переменные, введенные для приведения задачи к виду $(1)-(5)$, а $x_{10}, \ldots, x_{14}$ - переменные, введенные для обозначения левых частей отсечений. Первоначальная симплексная табл. 1 не является $l$-нормальной, но она приводится к нужному виду после одной симплексной итерации. Исходной для применения алгоритма служит табл. 2. Первая строка под табл. 2-6 - строка коэффициентов ограничения (13), вторая - строка коэффициентов отсечения 
Таблица 1

\begin{tabular}{l|r|rr}
\multicolumn{1}{c}{} & \multicolumn{1}{c}{1} & $-x_{1}$ & $-x_{2}$ \\
\cline { 2 - 3 }$x_{0}$ & 0 & -1 & -1 \\
\hline$x_{1}$ & 0 & -1 & 0 \\
$x_{2}$ & 0 & 0 & -1 \\
$x_{3}$ & 10 & $1 *$ & 1 \\
\cline { 2 - 3 }$x_{4}$ & 0 & -2 & 1 \\
$x_{5}$ & 2 & 1 & 0 \\
$x_{6}$ & 0 & -1 & 1 \\
$x_{7}$ & 4 & 1 & 0 \\
\hline$x_{3}$ & 0 & -1 & 2 \\
$x_{9}$ & 6 & 1 & 0 \\
\hline
\end{tabular}

Таблица 5

\begin{tabular}{l|r|rr}
\multicolumn{1}{c}{} & \multicolumn{1}{c}{1} & $-x_{3}$ & $-x_{12}$ \\
\cline { 2 - 3 }$x_{0}$ & 10 & 1 & 0 \\
\hline$x_{1}$ & 2 & -7 & 1 \\
$x_{2}$ & 8 & 8 & -1 \\
$x_{3}$ & 0 & -1 & 0 \\
\hline$x_{4}$ & -4 & -22 & 3 \\
$x_{5}$ & 0 & 7 & -1 \\
\hline$x_{6}$ & -6 & -15 & 2 \\
$x_{7}$ & 2 & 7 & -1 \\
\hline$x_{8}$ & -14 & -23 & 3 \\
$x_{9}$ & 7 & 7 & -1 \\
\hline
\end{tabular}

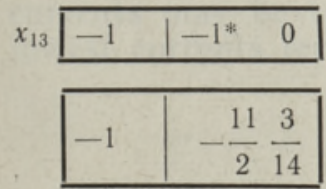

$\lambda=11 / 2$
Таблица 2 $\lambda=1 / 4$

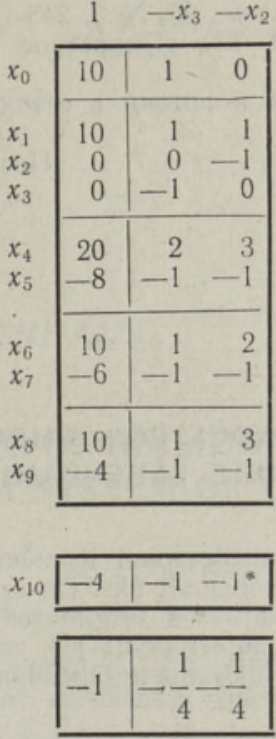

Таблица 3
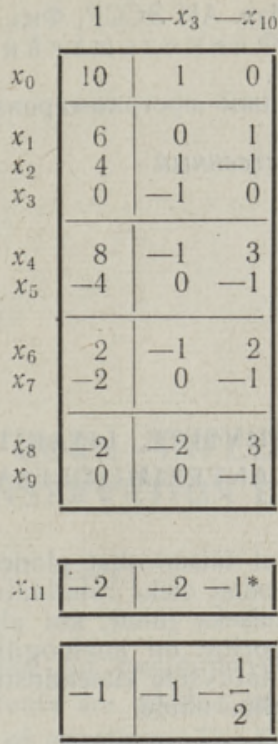

$\lambda=1 / 2$
Таблица 4

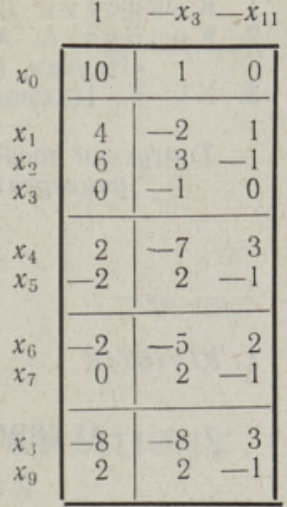

$-2 \mid-5-1^{*}$

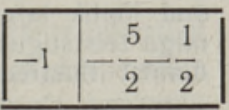

$\lambda=1 / 2$
Таблица 7

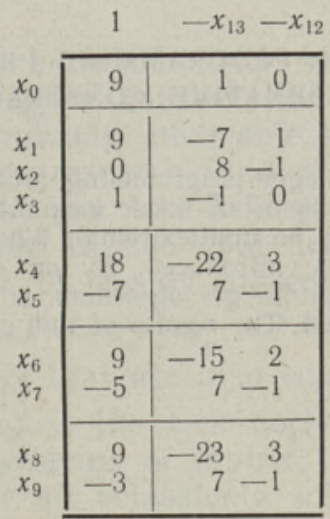

\begin{tabular}{|l|r|rr}
$x_{0}$ & \multicolumn{1}{c}{1} & $-x_{13}$ & $-x_{14}$ \\
\cline { 2 - 4 }$x_{1}$ & 9 & 1 & 0 \\
\hline$x_{2}$ & 6 & -4 & 1 \\
$x_{3}$ & 5 & 5 & -1 \\
1 & -1 & 0 \\
\hline$x_{4}$ & 3 & -13 & 3 \\
$x_{5}$ & -4 & 4 & -1 \\
\hline$x_{6}$ & 3 & -9 & 2 \\
$x_{7}$ & -2 & 4 & -1 \\
\hline$x_{8}$ & 0 & -14 & 3 \\
$x_{9}$ & 0 & 4 & -1 \\
\cline { 2 - 4 } & &
\end{tabular}

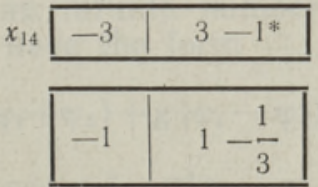

$\lambda=1 / 3$

(10), по которой строится отсечение (13). Ведущий элемент обозначен звездочкой. Из табл. 7 получается оптимальное решение примера: $x_{1}=6, x_{2}=3$ и оптимальное значение целевой функции $x_{0}=9$ (значения остальных вспомогательных переменных: $x_{3}=1, x_{4}=9, x_{5}=-4$, $\left.x_{6}=3, x_{7}=-2, x_{8}=x_{9}=0\right)$. Оптимальное решение удовлетворяет третьей группе альтернативных ограничений, 


\section{ЛИТ Е РА Т У РА}

1. Ки в и стик Л., Изв. АН ЭССР, Физ. Матем., 25, № 3, 245-250 (1976).

2. Корбут А. А., Финкельштейн Ю. Ю., Дискретное программирование, М., «Наука», 1969.

3. Х у Т., Целочисленное программирование и потоки в сетях, М., «Мир», 1974.

Тартуский государственный университет

L. KIVISTIK
Поступила в редакцию $30 / \mathrm{I} 1979$

\section{ALTERNATIIVSETE LISAKITSENDUSTEGA TAISARVULISTE PLANEERIMISULESANNETE LAHENDAMINE}

On vaadeldud lineaarset täisarvulist planeerimisülesannet lisanōudega, et mitmest antud lineaarvõrratuste süsteemist oleks rahuldatud vähemalt üks. On esitatud lihtne võte lōikekitsenduse konstrueerimiseks juhul, kui alternatiivsed tingimused pole rahuldatud. Saadud lōplik lahendusalgoritm on analoogiline autori poolt $\left.{ }^{[}{ }^{1}\right]$ varem avaldatud algoritmiga teistsuguste alternatiivsete kitsendustega ülesannete lahendamiseks. Algoritmi rakendamist illustreerib arvuline näide.

\section{KIVISTIK}

\section{THE SOLUTION OF INTEGER PROGRAMMING PROBLEMS WITH ADDITIONAL ALTERNATIVE CONSTRAINTS}

This paper considers a linear integer programming problem with the additional requirement that in a set of several systems of linear inequalities at least one system is satisfied. Generally this problem may be multiextremal. When the alternative constraints are not satisfied, legitimate cuts are introduced. By an «integrization» of these cuts an algorithm, similar to Gomory's all-integer algorithm, is obtained. Its finitness proof and a numerical example are presented. The results of this paper generalize author's previous results [']. 